\title{
Frequency Loss and Recovery in Rolling Bearing Fault Detection
}

\author{
Aijun $\mathrm{Hu}^{*}$, Ling Xiang, Sha Xu and Jianfeng Lin
}

\begin{abstract}
Rolling element bearings are key components of mechanical equipment. The bearing fault characteristics are affected by the interaction in the vibration signals. The low harmonics of the bearing characteristic frequencies cannot be usually observed in the Fourier spectrum. The frequency loss in the bearing vibration signal is presented through two independent experiments in this paper. The existence of frequency loss phenomenon in the low frequencies, side band frequencies and resonant frequencies and revealed. It is demonstrated that the lost frequencies are actually suppressed by the internal action in the bearing fault signal rather than the external interference. The amplitude and distribution of the spectrum are changed due to the interaction of the bearing fault signal. The interaction mechanism of bearing fault signal is revealed through theoretical and practical analysis. Based on mathematical morphology, a new method is provided to recover the lost frequencies. The multi-resonant response signal of the defective bearing are decomposed into low frequency and high frequency response, and the lost frequencies are recovered by the combination morphological filter (CMF). The effectiveness of the proposed method is validated on simulated and experimental data.
\end{abstract}

Keywords: Rolling element bearing, Signal processing, Frequency loss, Fault detection, Morphological filter

\section{Introduction}

Bearing defects are a common cause of machine breakdown. It is crucial to accurately diagnose the existence of rolling bearing fault. Vibration analysis is extensively employed rolling bearing fault detection. Many analysis methods have been proposed in the time domain $[1,2]$ and the frequency domain, such as demodulation algorithm [3, 4], cyclostationary analysis [5]. Time-frequency analysis techniques wavelet-transform (WT) [6, 7], EMD and LMD [8], for instance, are devoted to process bearing vibration signals as well.

When a rolling element moves over a damaged surface, it generates an impulsive force and excites resonances in the bearing and machine. The period of the impulses or characteristic frequency can be calculated, according to the rotating velocity, position of faults and bearing dimensions [9]. However, the bearing fault signals are almost always masked by background noise [10] and

*Correspondence: bdlaohu@126.com

Department of Mechanical Engineering, North China Electric Power University, Baoding 071003, China the defect frequency identification from direct vibration signals becomes difficult. Randall [11] illustrated that the low harmonics of the bearing characteristic frequencies are almost invariably strongly masked by other vibration components. It is difficult to identify the bearing fault in the spectrum using conventional FFT methods.

In the current literature, although the low harmonics of the bearing characteristic frequencies [11] and the shaft-speed frequency [12] can sometimes be found in raw spectrum, it is widely recognized that low frequency components of bearing fault signal cannot be usually observed in the spectrum. Here, we define the phenomenon that the low frequency components cannot be observed in the FFT spectrum as frequency loss. The low frequency components include bearing characteristic defect frequencies, rotating frequency and their harmonics. As for the causes of the frequency loss, the explanation in published literatures is summarized (i) masked by the strong background noise [13], (ii) masked by the interference vibrations from other machine elements [11, 14], (iii) may not exist at all in the measured signal sometimes [15]. 
However, frequency loss can be observed when the bearing is running under the condition of low background noise and no other vibration components existing. Moreover, if the lost frequencies are masked by background noise, the low frequency band in the spectrum must present the feature of noise, but sometimes there is no such noise feature when the frequency loss occurs. In addition, it is noticed in the gear/bearing model study that the gearmesh frequency is modulated at both the shaft speed and the bearing characteristic frequency [16]. Obviously, if the lost frequencies are masked by other vibration components, features of the external vibration components and the action between the characteristic frequency and the external vibration should be found. It does not mean that the low frequency components cannot be observed. Furthermore, frequency loss is also observed in the sideband frequencies and the resonant frequencies in the paper. The features of the spectrum are used in the calculation of spectral kurtosis [14], correlated kurtosis [17] and entropy [18, 19] for the central frequency and band width selection in the bearing fault detection. Obviously, the existence of frequency loss will affect the calculation results of these indicators. Study on the root cause of the frequency loss in bearing fault signal is helpful to obtain a better understanding of the bearing fault feature and its detection.

This paper discusses the interaction of the bearing vibration signal. It is demonstrated that the phenomenon of the frequency loss is generated by the internal vibrations rather than the external interference. The interaction mechanism of the bearing signal is revealed through theoretical and practical analysis. A new method based on morphological filter (MF) is proposed to recover the lost frequencies in bearing fault signal.

The paper is organized as follows. The phenomenon of frequency loss in the bearing vibration signal is presented through experiments in Section 2. The interaction mechanism of the bearing signal is explained in Section 3. A novel method is presented to recover the lost frequencies based on morphological filter. And the proposed technique is evaluated using the simulation and experimental signals in Section 4. Some remarks and conclusions are drawn in Section 5.

\section{Frequency Loss in the Bearing Vibration Signal}

In this section, the phenomenon of frequency loss in the bearing vibration signal is presented through two independent experiments. It is demonstrated that the frequency loss can be observed even the bearing is running under the condition of low background noise and no other vibration components existing.

Two different types of bearing, N205 cylindrical roller bearing and SKF 6205 deep groove ball bearing are employed in the experiments. The calculated bearing defect characteristic frequencies [9], ball pass frequency of the outer race (BPFO) and ball pass frequency of the inner race (BPFI) of the two experimental bearings are given in Table 1 .

\subsection{Experiment 1: Vibration Signal of Bearing with Severe Defect}

The first experiment is carried out on a bearing and gearbox fault test rig (Figure 1(a)) at the Vibration Lab at North China Electric Power University (NCEPU). The test rig consists of a motor (left), support bearing (center) and a spring loader (right). The test bearing is located at the right end of the shaft. A slot defect (0.6 $\mathrm{mm}$ in width and $0.3 \mathrm{~mm}$ in depth) on the outer and inner race are introduced to the test bearings (N205) using electric discharge machining, as shown in Figure 1(b). Vibration data is collected using accelerometers, which are placed on the vertical and horizontal position of the test bearing housing. A radial load is added to the shaft and bearing by a spring mechanism.

During the data acquisition, the shaft rotating speed is kept around $1440 \mathrm{r} / \mathrm{min}(24 \mathrm{~Hz})$. The sampling frequency is set to $12.8 \mathrm{kHz}$. The time waveform, spectrum and envelop spectrum of raw outer and inner race fault vibration signal of bearing N205 is provided in Figure 2 and Figure 3. Considering the relative large defect size in the outer and inner race, the defect is classified as severe. Figure 2 and Figure 3 show that strong transient impulses are generated in the time waveform. The excited resonances in the spectrum are clear and have relative high amplitude. The envelope analysis, Figure 4 indicates that the rotational speed frequency, BPFO, BPFI and their harmonics can be easily singled out. The side band of BPFI is clear in the envelop spectrum (Figure 4(b)). However, the low frequencies, such as rotational speed frequency, BPFO or BPFI are not visible in the spectrum. Furthermore, the amplitude of most of the components in the low frequency region is almost zero, which means the background noise is very low during the data acquisition. It is evident that the almost zero amplitude of BPFO or BPFI in the spectrum is incompatible with the high amplitude of the impulses in the time waveform.

Table 1 Characteristic frequencies

\begin{tabular}{lll}
\hline Bearing type & BPFO $(\mathbf{H z})$ & BPFI (Hz) \\
\hline N205 & $116.0($ at $1440 \mathrm{r} / \mathrm{min})$ & $172.0($ at $1440 \mathrm{r} / \mathrm{min})$ \\
6205 & $107.7($ at $1797 \mathrm{r} / \mathrm{min})$ & $159.9($ at $1772 \mathrm{r} / \mathrm{min})$ \\
\hline
\end{tabular}




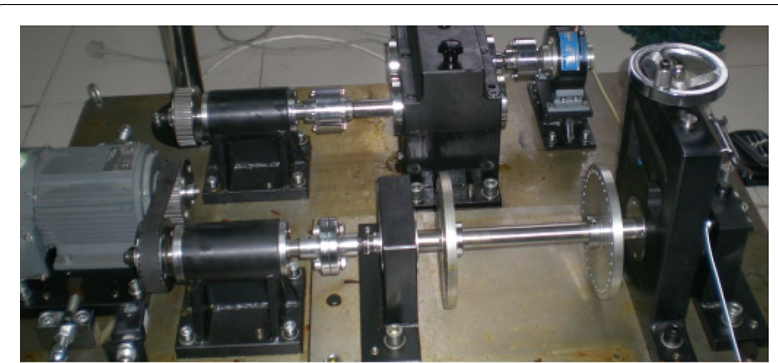

$\mathbf{a}$

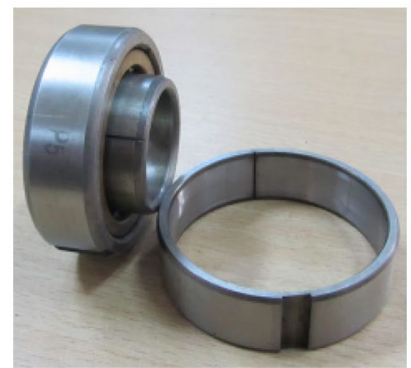

b

Figure 1 (a) Bearing and gearbox fault test rig, (b) Outer race and inner race defective bearing

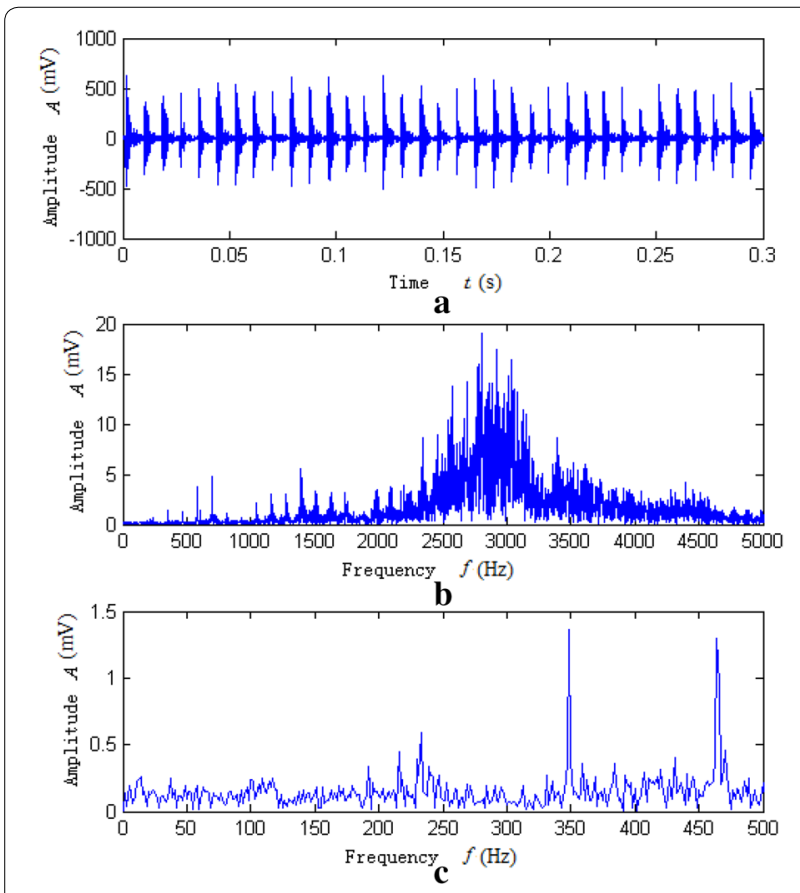

Figure 2 Outer race fault signal of N205: (a) time waveform, (b) FFT spectrum, (c) local spectrum

\subsection{Experiment 2: Vibration Signal of Bearing with Slight Defect}

The second experimental data of rolling bearing are collected from the CWRU Bearing Data Centre Website [20]. In this experiment, single point faults are seeded
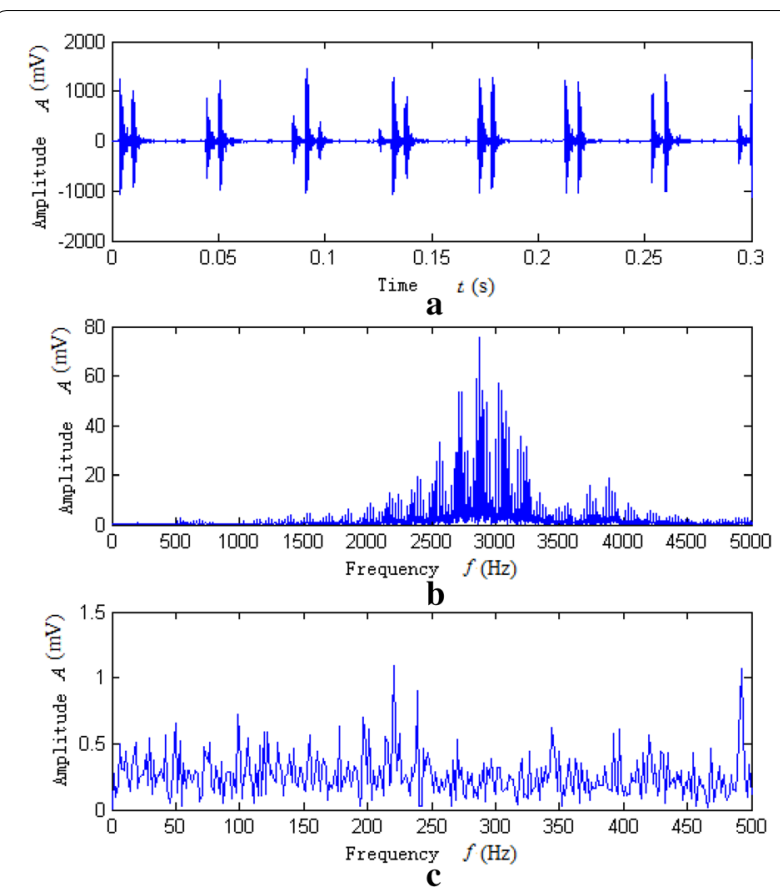

Figure 3 Inner race fault signal of N205: (a) time waveform, (b) FFT spectrum, (c) local spectrum

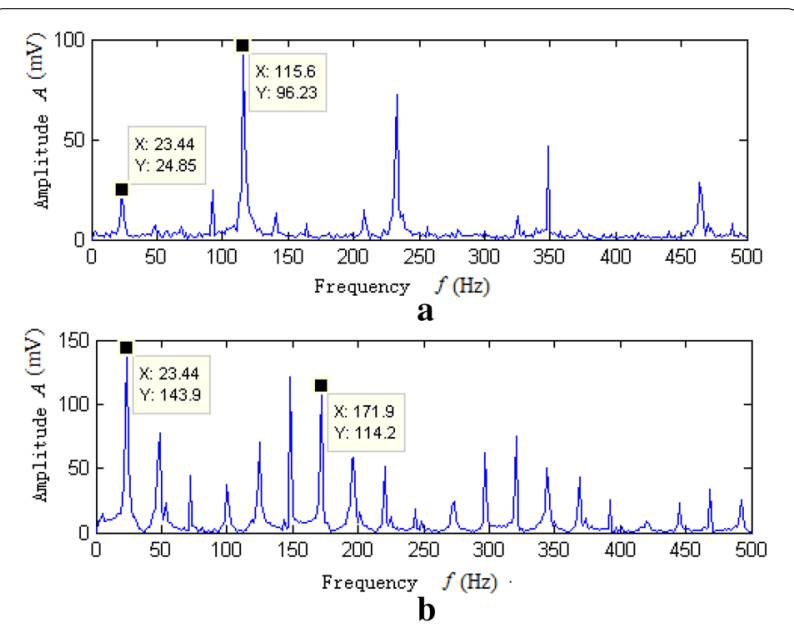

Figure 4 Envelope spectrum of N205: (a) outer race defect, (b) inner race defect

to the test bearing with different fault diameters. More detail about the experiment condition can be found in the Website. The measurement is performed with sampling frequency of $12 \mathrm{kHz}$. Vibration data of fault size $0.177 \mathrm{~mm}(0.07 \mathrm{in})$ in outer race and inner race are selected in the paper. The $0.177 \mathrm{~mm}$ is the smallest fault size in this experiment. The defect is classified as slight. 

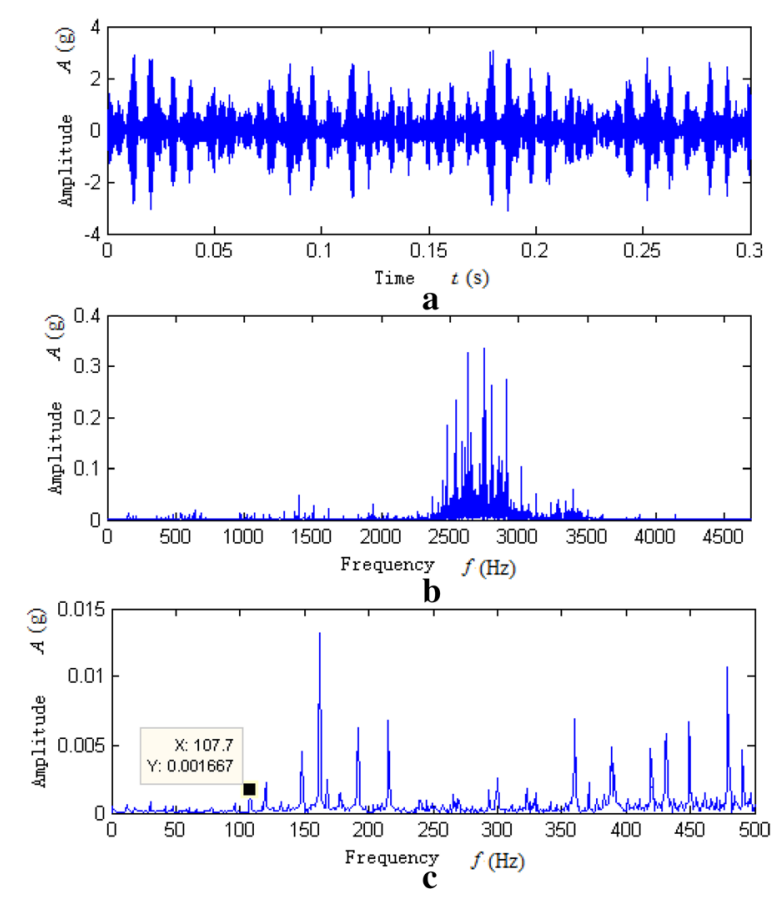

Figure 5 Outer race fault signal of 6205: (a) time waveform, (b) FFT spectrum, (c) local spectrum

The outer race and inner race fault signal of 6205 is shown in Figure 5. Figure 5(c) shows that the outer race fault characteristic frequency BPFO can be found in the spectrum with very low amplitude, and the rotational frequency is not visible. For the inner race fault, BPFI should be modulated at the shaft speed (Fr). The characteristic frequency BPFI is presented in Figure 6(c), but the side frequencies are not indistinctive, particularly the left side frequency BPFI-Fr is almost zero.

The phenomenon of frequency loss is observed in the two independent experiments. Comparing the two experiments, some common features can be concluded: (1) the experiments are carried out in the laboratory, where has low background noise. (2) Only the test rigs are running, no external vibration interference. It is demonstrated that background noise or external interference cannot be the root cause of the frequency loss. Furthermore, various fault degree leads to contrary result in the experiments. All of the low frequency components are lost in the severe bearing fault test (N205) and the low frequency components are partly lost in the slight bearing fault test (6205). The cause of frequency loss must be the result of internal action in the bearing fault signal.
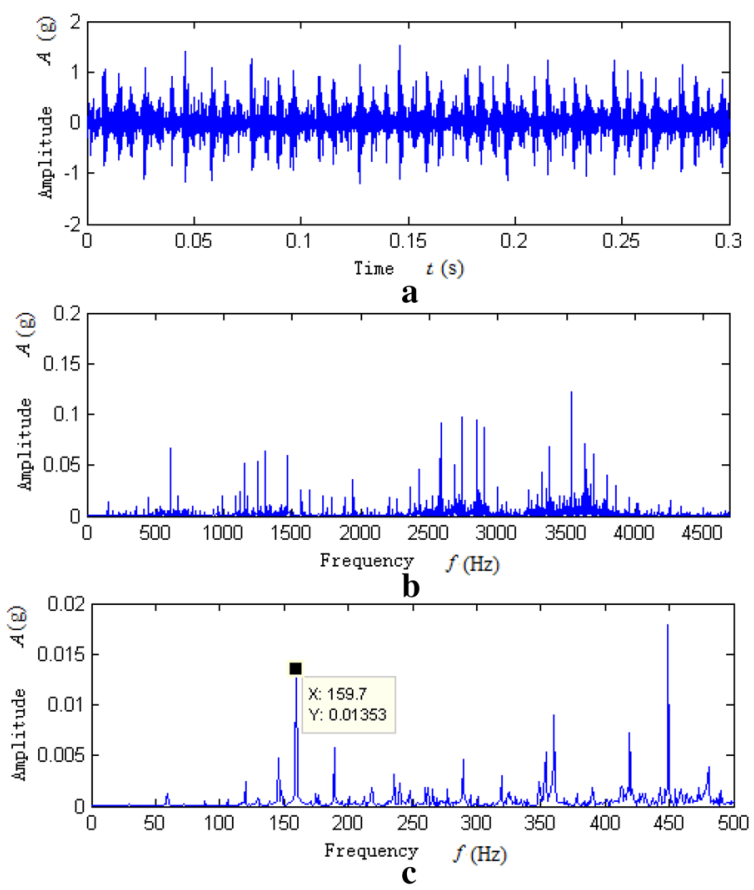

Figure 6 Inner race fault signal of 6205: (a) time waveform, (b) FFT spectrum, (c) local spectrum

\section{Interaction Mechanism of the Bearing Signal}

In this section, the interaction of the bearing fault signal is discussed using the simulated signal. The repetitive impact signal from a localized bearing defect can be described by a train of Dirac delta function $\delta(t)$ with the period between two successive impulses being $T_{d}$ :

$$
x(t)=\sum_{k=0}^{N} A_{k} \delta\left(t-k T_{d}\right),
$$

where $T_{d}$ is the reciprocal of the characteristic frequency, and $A_{k}$ is the amplitude of the impacts.

The impulsive response from the bearing faults can be expressed as:

$$
s(t)=B e^{-\xi t} \cos \left(2 \pi f_{n} t+\phi_{n}\right),
$$

where $B$ denotes the amplitude of the impulsive response, $f_{n}$ is the excited natural frequency, $\phi_{n}$ is the initial phase angle, $\xi$ is the attenuation factor, for a single degree of freedom (SDOF), $\xi=\alpha 2 \pi f_{n}$, where $\alpha$ denotes the relative damping ratio.

Ignoring the influence of roller slippage, the vibration signal arising from a localized fault in a bearing can be expressed by 


$$
y(t)=\sum_{k=0}^{N}\left[A_{k} \delta\left(t-k T_{d}\right) \otimes s(t)\right],
$$

where the symbol $\otimes$ denotes convolution.

From the above experimental cases, multiple resonant frequency bands are found in the spectrum, i.e., frequency bands about $1500 \mathrm{~Hz}$ and $2800 \mathrm{~Hz}$ in Figure 2(b), $2800 \mathrm{~Hz}$ and $4000 \mathrm{~Hz}$ in Figure 2(c), $1200 \mathrm{~Hz}$, $2700 \mathrm{~Hz}$ and $3600 \mathrm{~Hz}$ in Figure 3. Therefore, the actual bearing fault signal is the cumulating of multiple impulse responses with different resonant frequencies. The transmission path of different resonant responses to the transducer varies to different components. So the collected vibration signal is multi-resonant responses with time difference. Two impulse responses are also reported in the vibration signal of a spalled bearing when the rolling element entry into and exit from the spall [21].

In the following sections, frequency domain analysis (amplitude and phase) of the simulated signal in Eq. (3) is performed and then a dual-resonant-frequency bearing fault signal is used to discuss the interaction in the bearing fault signal. The single side Fourier transform of Eq. (1) can be expressed as

$$
F T[x(t)]=2 A_{k} / T_{d} \sum_{k=0}^{N} \delta\left(f-k f_{d}\right),
$$

where $f_{d}=1 / T_{d}, F T[\cdot]$ denotes the Fourier transform.

The frequency domain expression of the impulsive response is

$$
F T[s(t)]=A\left(f-f_{n}\right) e^{j\left[\phi\left(f-f_{n}\right)+\phi_{n}\right]},
$$

where the amplitude $A(f)=\frac{B}{\sqrt{\xi^{2}+(2 \pi f)^{2}}}$, the phase $\phi(f)=-\arctan (2 \pi f / \xi)$, it is known that $\phi(f) \rightarrow \pi / 2$ when $f \rightarrow+\infty$ and $\phi(f) \rightarrow-\pi / 2$ when $f \rightarrow-\infty$.

The final expression of Eq. (3) in frequency domain is

$$
\begin{aligned}
& Y(f)=F T[y(t)] \\
& =\frac{2 A_{k}}{T_{d}} \sum_{k=0}^{N} A\left(f-k f_{d}-f_{n}\right) e^{j\left[\phi\left(f-k f_{d}-f_{n}\right)+\phi_{n}\right]} .
\end{aligned}
$$

Thus, $Y(f)$ has a discrete spectrum, and the corresponding phase is the sum of $\phi(f)$ and $\phi_{n}$.

Two different resonant responses excited by the same period impact are simulated. The sampling frequency is set to $8192 \mathrm{~Hz}, T_{d}$ is set to 128 sample points, the corresponding characteristic frequency is $64 \mathrm{~Hz}$. The amplitude of the impulse forces and the impulse responses are both set to 1 , the attenuation factor $\xi$ is equal to 600 , and total 8192 samples are used for further analysis. The natural frequency is set to $1000 \mathrm{~Hz}$ (response one) and $2000 \mathrm{~Hz}$ (response two), respectively. Figure 7 and Figure 8 depict the two simulated responses with $0^{\circ}$ initial phases. From the spectra, the two signals have same

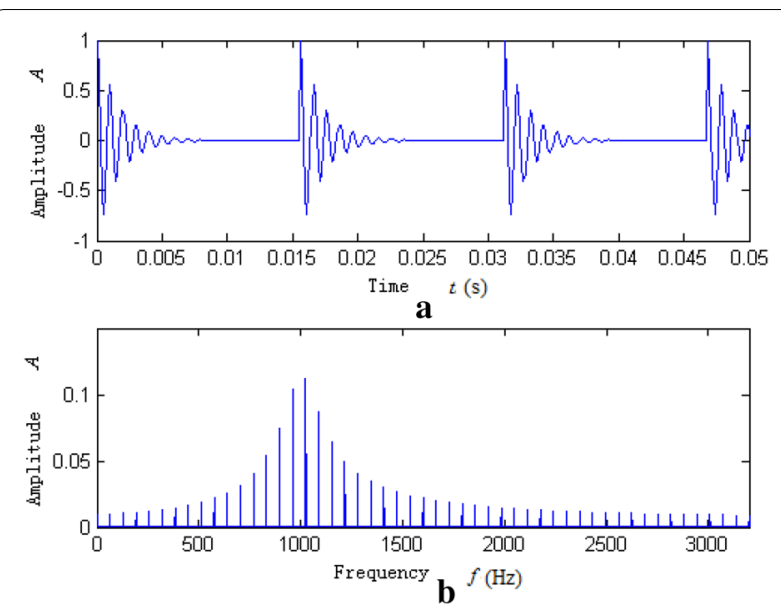

Figure $\mathbf{7}$ Response 1 (natural frequency: $1000 \mathrm{~Hz}$, phase: $0^{\circ}$ ): (a) time waveform, (b) FFT spectrum

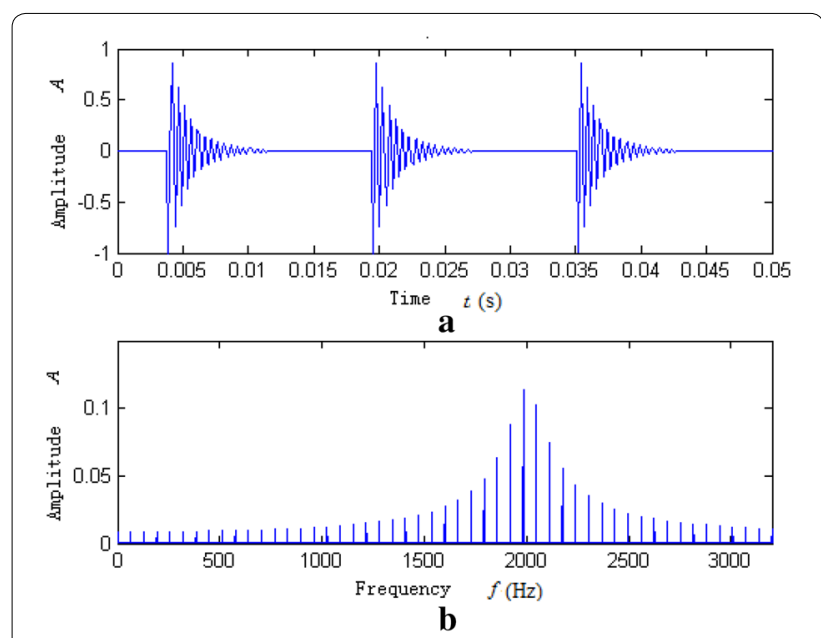

Figure 8 Response 2 (natural frequency: $2000 \mathrm{~Hz}$, phase: $0^{\circ}$ ): (a) time waveform, (b) FFT spectrum

characteristic frequency and the amplitude of the low frequency components ( $64 \mathrm{~Hz}$ and its low order harmonics) is approximately equal. The spectrum lines are symmetrical relative to the central (resonant) frequency.

The parameters of the first response remains constant, the second response is superposed under different conditions. Superposition conditions are listed in Table 2. Superposition results are provided in Figures 9, 10, 11, 12. Compared to the original response (Figure 7 and Figure 8), the superposition results indicated that the spectrum varies greatly due to the different superposition conditions. As Figure 10(b) shown, superposition result under condition 2 illustrates that the characteristic frequency $(64 \mathrm{~Hz})$ and its low order harmonics can hardly be found in the spectrum. The frequency loss 
Table 2 Superposition conditions

\begin{tabular}{lll}
\hline No. & \multicolumn{2}{l}{ Response 2 } \\
\cline { 2 - 3 } & Initial phase & Start position \\
\hline Condition 1 & $0^{\circ}$ & 0 \\
Condition 2 & $-180^{\circ}$ & 0 \\
Condition 3 & $0^{\circ}$ & Point 32 \\
Condition 4 & $-180^{\circ}$ & Point 32 \\
\hline
\end{tabular}

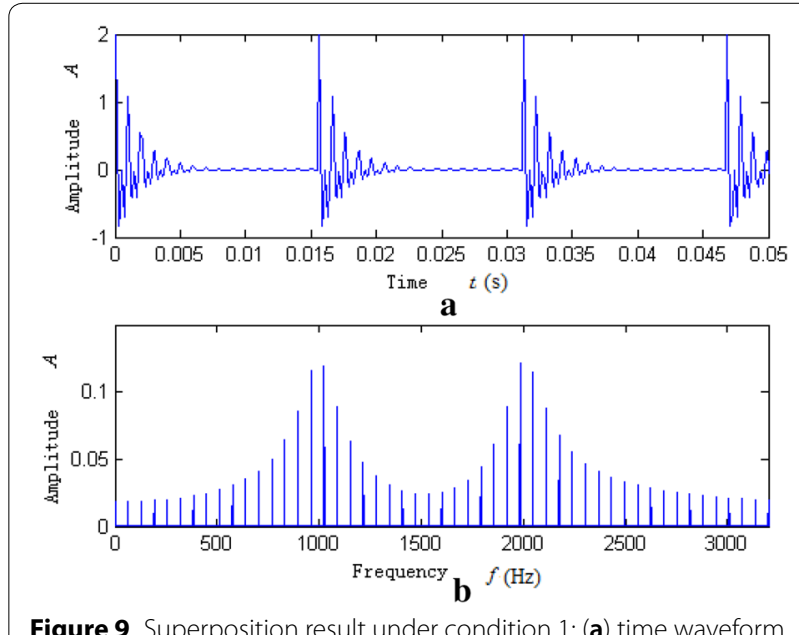

Figure 9 Superposition result under condition 1: (a) time waveform, (b) FFT spectrum

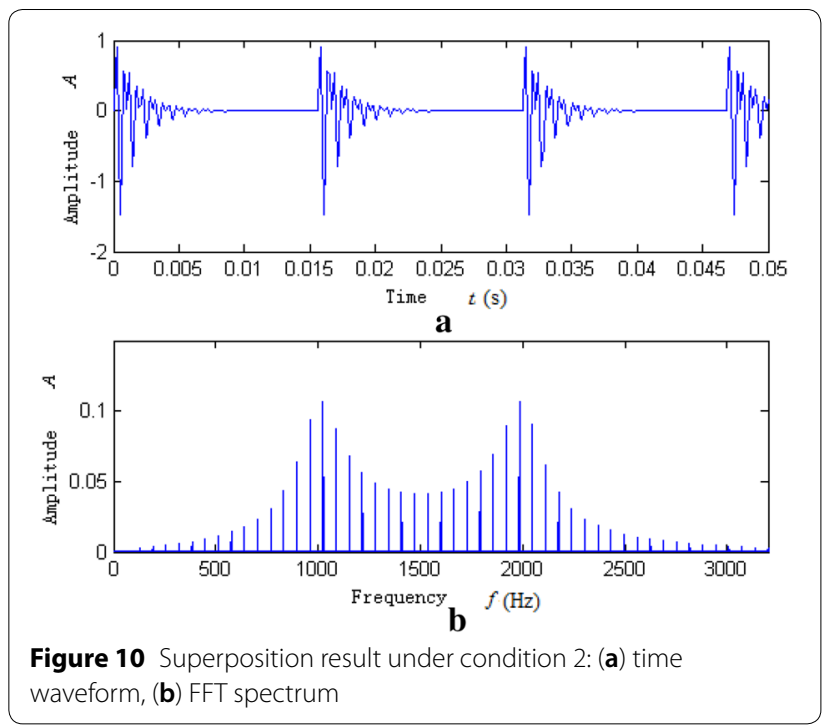

phenomenon is observed in the simulation when the two responses have an opposite phase. At the same time, the amplitude of the peaks around $1500 \mathrm{~Hz}$ is doubles that of the original response. From the superposition result
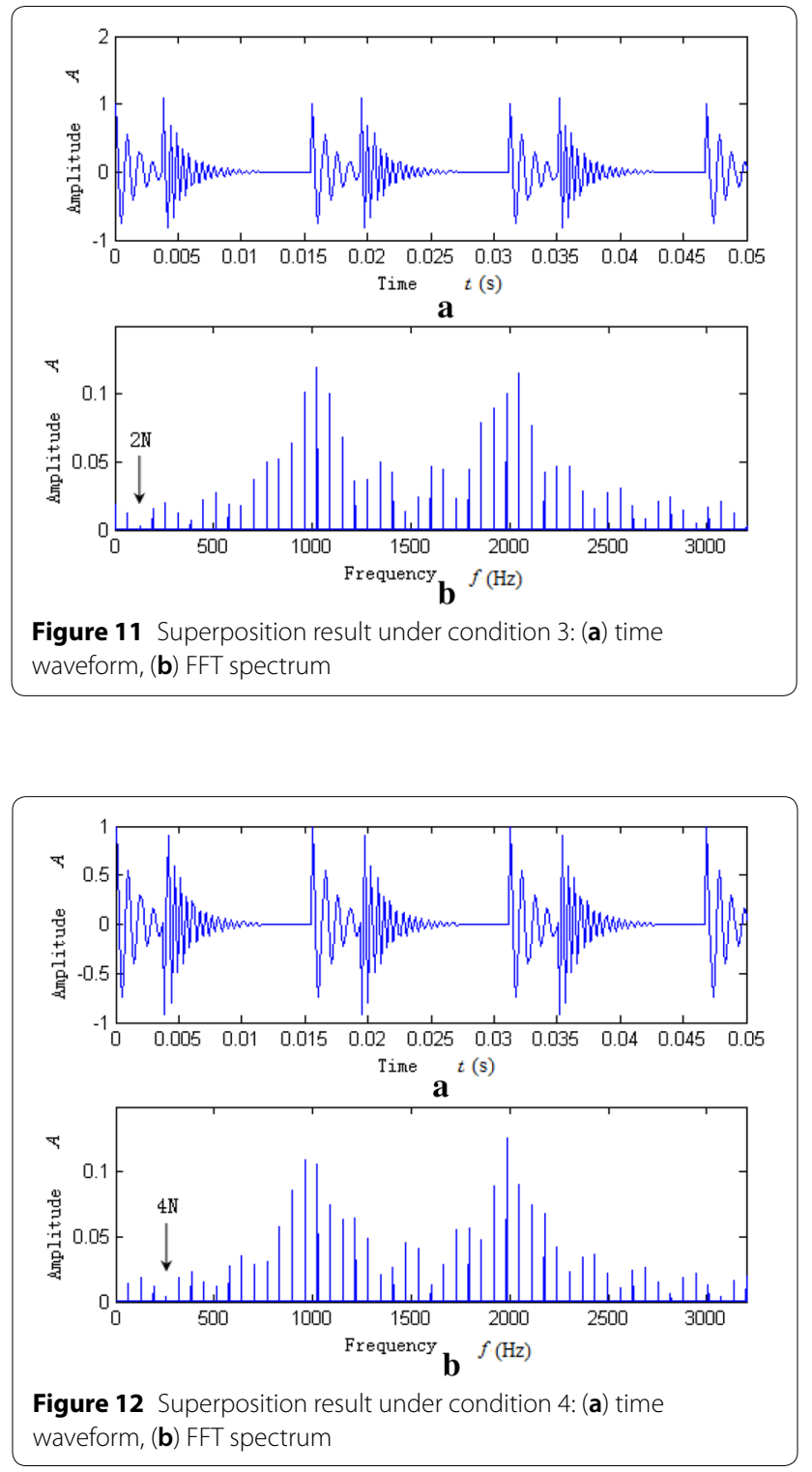

under condition 1, the amplitude of the low frequency components in Figure 9(b) is approximately twice as that of the original response, and the amplitude of the peaks around $1500 \mathrm{~Hz}$ is almost equal to its original value.

Theoretical calculation using Eq. (6) indicates that the amplitude of the superposed response in the spectrum is the vector sum of that of each response. From Eq. (5), the phase of the two exponential decay functions approach $-90^{\circ}$ at the low frequency band. When the initial phase $\phi_{n}$ is set to zero (condition 1), the final phase of the two responses is almost same (approximately $-90^{\circ}$ ). Therefore, the phase difference of the two responses at the low frequency band is almost $0^{\circ}$, and the amplitude of the superposed response is the summation of that of the each response. Similarly, when the initial phase of the second 
response is set to $-180^{\circ}$ (condition 2), the phase difference of the two responses is $-180^{\circ}$, and the amplitude of the two responses is cancelled out each other. So the frequency loss is observed under the superposition condition two.

Figure 11 and Figure 12 are the superposition results when the start position of the second response is right shifted 32 points. Some harmonics, the second order harmonic $(2 \mathrm{~N})$ of the characteristic frequency in Figure 11(b) and $4 \mathrm{~N}$ in Figure 12(b), for instance, are almost invisible. Other harmonics, including the resonant frequencies and their sideband frequencies, are changed obviously.

The influence of the start position in response two can be easily explained by the time-shifting property of the Fourier transform:

$$
\text { If } x(t) \leftrightarrow X(f) \text { then } x\left(t-t_{0}\right) \leftrightarrow X(f) e^{-j 2 \pi f t_{0}},
$$

where $X(f)$ is the Fourier transform of $x(t), t_{0}$ is the time delay.

The second response starts at 32 point, which amounts to a quarter of the period, the phase delays of the first four harmonics are calculated as $-\pi / 2,-\pi, \pi / 2,0$. Due to the time-shifting of the second response, a total $-180^{\circ}$ phase difference is produced between the two responses at $2 \mathrm{~N}$ (Figure 11(b)) under condition three and at $4 \mathrm{~N}$ (Figure 12(b)) under condition 4.

According to the theoretical calculation and simulation, the interaction mechanism of the defective bearing signal is summarized. When rolling elements strike a local fault on the inner or outer race a shock is introduced that excites high frequency resonances of the whole structure between the bearing and the transducer. The collected vibration signal of the defective bearing consists of multi-resonant response. The final amplitude of the harmonics in the spectrum is the vector sum of that of each response. The different responses can cancel out each other when the phase difference between the responses is equal to $-180^{\circ}$. The interaction of the multiresonant response leads to frequency loss and distortion in the Fourier transform spectrum. Therefore frequency loss can be found in the low frequency harmonic, the sideband frequency and the resonant frequency.

\section{Recovery the Lost Frequencies Based on Mathematical Filter}

The root cause of frequency loss in the defective bearing signal is the interaction of the multi-resonant response. Therefore the key point to recover the lost frequencies depends on the separation of the different resonant response.
Mathematical morphology (MM) [22, 23] is a kind of nonlinear analysis method which was first used in image analysis. Unlike the traditional frequency domain filter, the morphological filter (MF) decomposes the signal into several physical components according to the signal geometric characteristics. The morphological filters have been adopted for fault feature extraction of bearing vibration signal [24-26].

\subsection{Morphological Filter in Brief}

All the MM processing is based on two basic operators, dilation and erosion. Let $f(n)$ and $g(m)$ be the onedimensional discrete signal and structural element (SE) respectively. The erosion of $f(n)$ by $g(m)$, denoted by $(f \Theta g)$ is defined as:

$$
\begin{aligned}
& (f \Theta g)(n)=\min [f(n+m)-g(m)], \\
& m \in 0,1, \ldots, M-1 .
\end{aligned}
$$

Dilation is defined as:

$$
\begin{aligned}
& (f \oplus g)(n)=\max [f(n-m)+g(m)], \\
& m \in 0,1, \ldots, M-1 .
\end{aligned}
$$

Based on dilation and erosion, two other basic morphological operators, the closing and the opening can be further defined. The opening is defined as a dilation of the eroded $(f \Theta g)$ signal by $g$ :

$$
(f \circ g)(n)=((f \Theta g) \oplus g)(n) .
$$

The closing is defined as an erosion of the dilated $(f \oplus g)$ signal

$$
(f \cdot g)(n)=((f \oplus g) \Theta g)(n) .
$$

The positive and negative impulses can be smoothed by closing and opening operations, respectively. To restrain both positive and negative impulse, the opening-closing $(\mathrm{OC})$ and closing-opening (CO) filters are composed through proper calculation combination, defined as

$$
\begin{aligned}
& F_{O C}(f(n))=((f \circ g) \cdot g)(n), \\
& F_{C O}(f(n))=((f \cdot g) \circ g)(n) .
\end{aligned}
$$

Although the combination of opening and closing can filter positive and negative impulse noise at the same time, statistic bias is existed. The output magnitude of the OC becomes small due to the opening operator's expansibility and closing operator's contractibility. On the contrary, the output magnitude of the $\mathrm{CO}$ is large. Therefore the average value of the two operators, namely combination morphological filter (CMF), is defined as

$$
y(n)=\frac{1}{2}\left[F_{C O}(f(n))+F_{O C}(f(n))\right] .
$$




\subsection{Property Morphological Filter}

Based on our previous analysis of working mechanism of mathematical morphological operators, the CMF is employed to recover the lost frequencies in the defective bearing signal. More details of the properties of different morphological operators, frequency response, selection principle of SE length can be found in Ref. [27]. The CMF presents low pass filter property. The high frequency component can be extracted by subtracting the CMF output from the original signal. Therefore, the multiresonant response can be decomposed into two parts after the CMF processing, low frequency and high frequency response. Once the multiresonant response is separated, the effect of the interaction is eliminated and the lost frequencies are visible in the spectrum.

The property of CMF in impulsive type signal processing is analyzed firstly. The traditional digital filters, finite impulse response filter (FIR), for instance, has been adopted to compose the filter bank in fast kurtogram (FK) algorithm [28] and high-resolution spectral analysis [29]. The FIR filter with a standard Kaiser window [29] and the CMF are taken to process the same signal for the comparison study. A $50 \mathrm{~Hz}$ sinusoid signal with 0.1 amplitude is superposed to the impulsive response 2 . The time waveform and spectrum of the simulated signal are shown in Figure 13. The CMF is used to filter the simulated signal. The SE length is set to 3 , and the corresponding cutoff frequency is $987 \mathrm{~Hz}$ [27]. From the filter result (Figure 14), the low frequency component (sinusoid signal) is well preserved after CMF processing and the phase delay is 0 . The high frequency component (impulsive response) is extracted by subtracting the CMF output from the original signal, which is shown in Figure 15. It shows that the time waveform and the spectrum of the extracted signal are almost the same as the original simulated signal, thus the impulsive responses are well extracted by CMF.

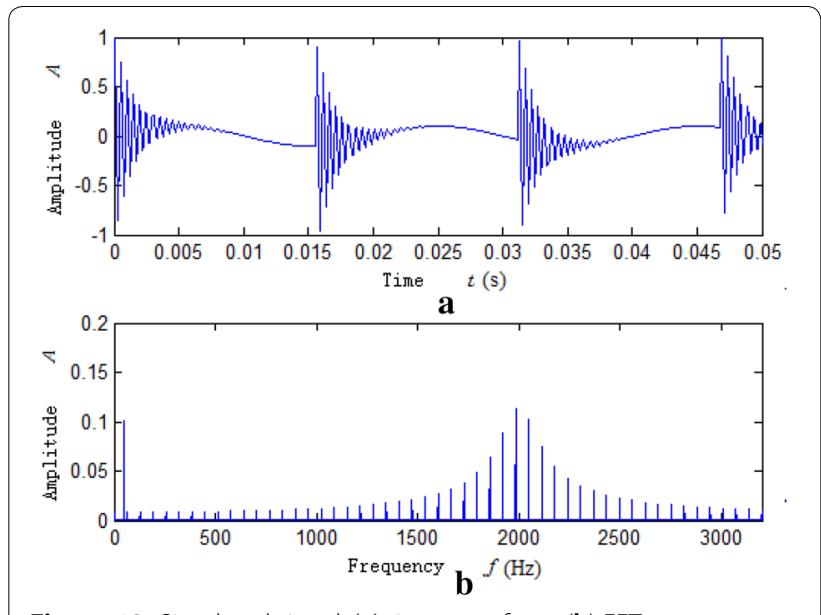

Figure 13 Simulated signal: (a) time waveform, (b) FFT spectrum

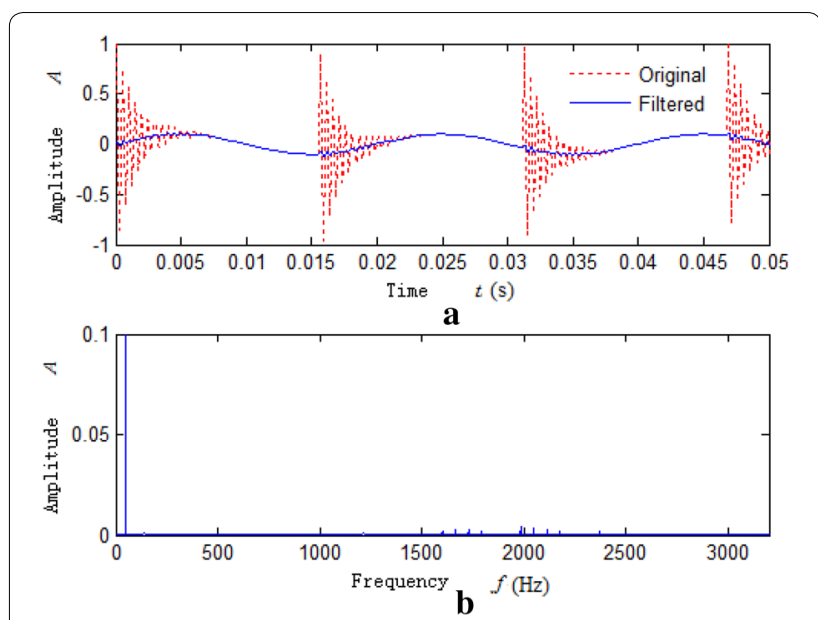

Figure 14 Filter result of the CMF: (a) time waveform, (b) FFT spectrum

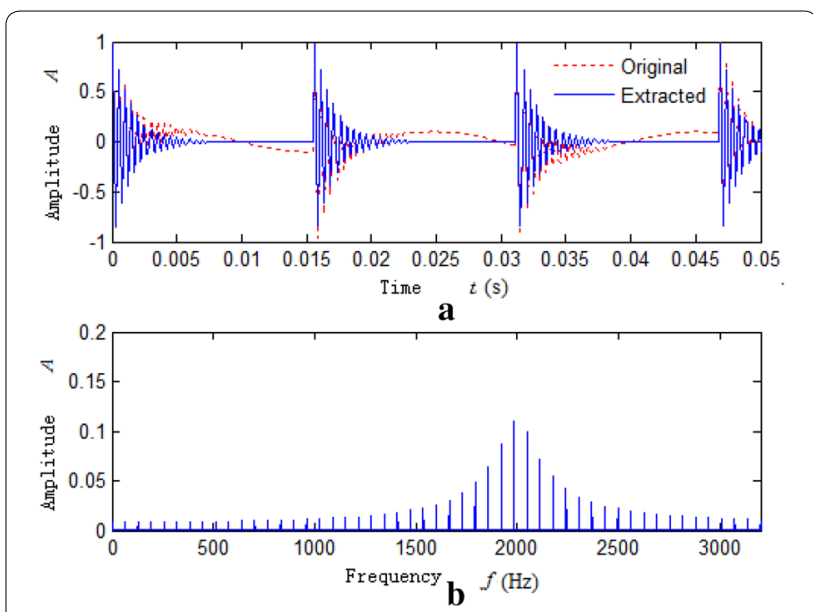

Figure 15 Extraction result of the CMF: (a) time waveform, (b) FFT spectrum

The low pass FIR and high pass FIR are used to process the simulated signal. The cutoff frequency of the FIR is set to $1000 \mathrm{~Hz}$, which is similar to that of the CMF. The results of low pass FIR process, Figure 16 and Figure 17, show that the residual components (indicated as point A in Figure 16) of the impulsive response signal are preserved after low pass FIR processing. These residual components are presented as discrete harmonics in the spectrum. The high pass FIR is adopted to extract the impulsive response signal. It is shown that distortion (point B) at the beginning of the impulsive response is found in Figure 17. Therefore only part of the distributional spectrum can be filtered or extracted by FIR. The impulsive responses cannot be completely recovered by the frequency-domain based (low-pass, band-pass or high-pass) filter, unless the whole frequency band is 


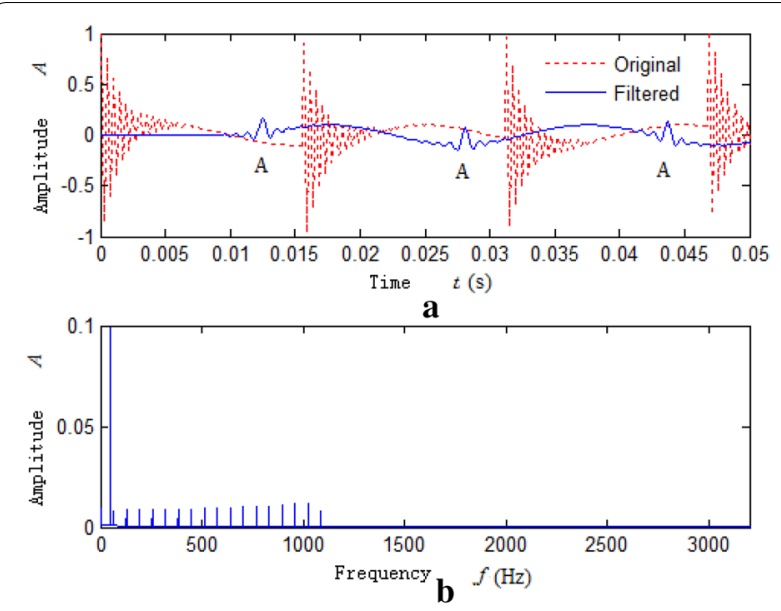

Figure 16 Filter result of the low pass FIR: (a) time waveform, (b) FFT spectrum

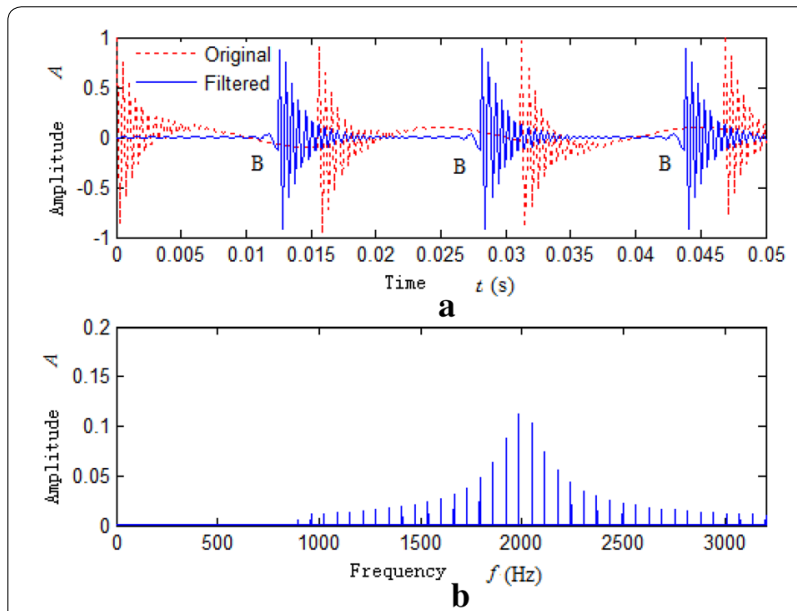

Figure 17 Filter result of the high pass FIR: (a) time waveform, (b) FFT spectrum

taken as the filter band. On the contrary, the CMF processes the signal according to the signal geometric characteristics rather than the frequency distribution. The part of the waveform whose width is narrow than the SE length can be filtered or extracted by CMF processing. The different impulsive type signals can be separated according to different geometric scale.

\subsection{Recovery the Lost Frequencies in Bearing Fault Signal Based on CMF}

The above mentioned experimental data of bearing N205 and 6205 is processed by the CMF to verify the lost frequencies recovery property of morphological filter in real defective bearing signal. The signal of N205 with severe defect on the outer race is decomposed into the low frequency and the high frequency component after CMF processing, which are shown in Figure 18 and Figure 19. The lost frequencies, characteristic frequency (BPFO),

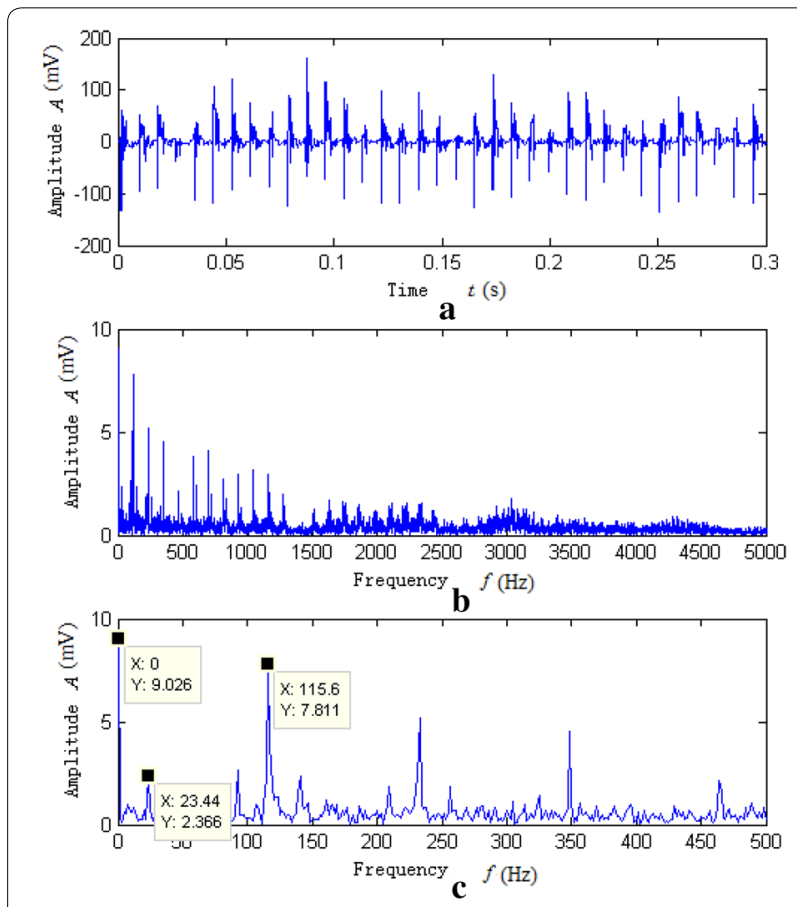

Figure 18 Low frequency component of N205 with severe defect on the outer race: (a) time waveform, (b) FFT spectrum, (c) local spectrum

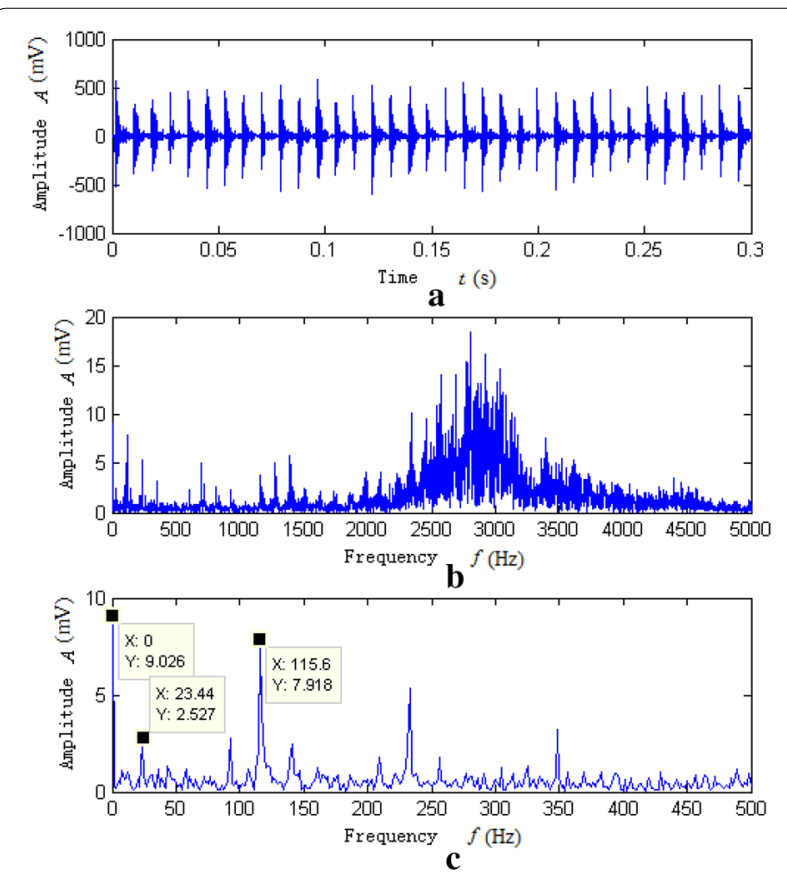

Figure 19 High frequency component of N205 with severe defect on the outer race: (a) time waveform, (b) FFT spectrum, (c) local spectrum 


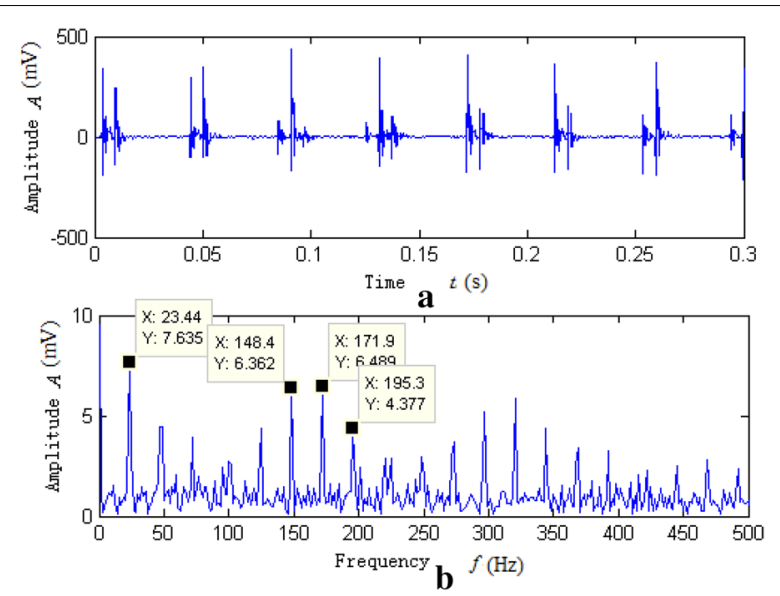

Figure $\mathbf{2 0}$ Low frequency component of N205 with the severe defect on the inner race: (a) time waveform, (b) local spectrum

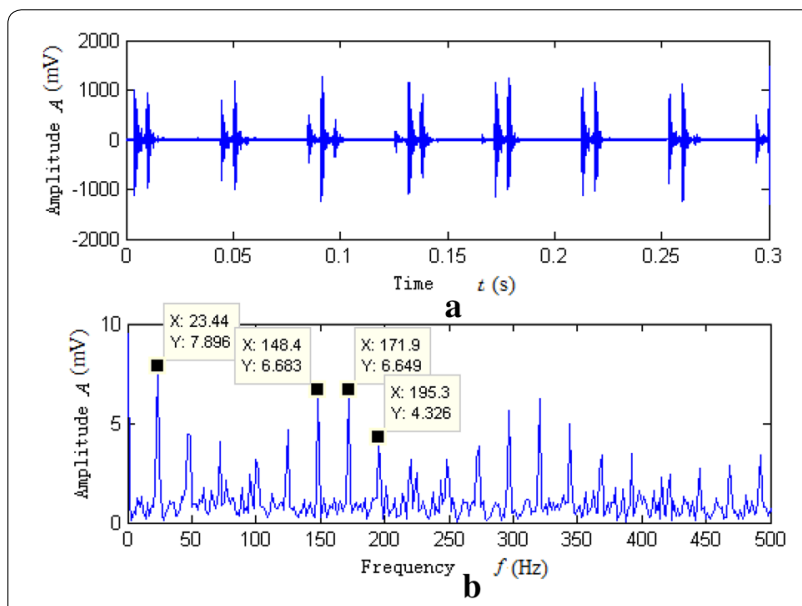

Figure 21 High frequency component of N205 with the severe defect on the inner race: (a) time waveform, (b) local spectrum

rotational speed frequency are recovered both in the low and high frequency component. The harmonic at $0 \mathrm{~Hz}$ in Figure 18(c) and Figure 19(c), which is not observed in the original spectrum, is produced by the period impulse response. It supplies another evidence of the validity of the proposed method.

Figure 20 and Figure 21 depict the CMF processing result of the severe inner race defect signal of N205. The lost frequencies, characteristic frequency (BPFI) and its sidebands, rotational speed frequency and its harmonics are recovered. The modulation with the shaft speed property of the bearing inner race fault is clearly presented in the spectrum.

The CMF is applied to the experimental data collecting from the CWRU and the results are shown in Figure 22

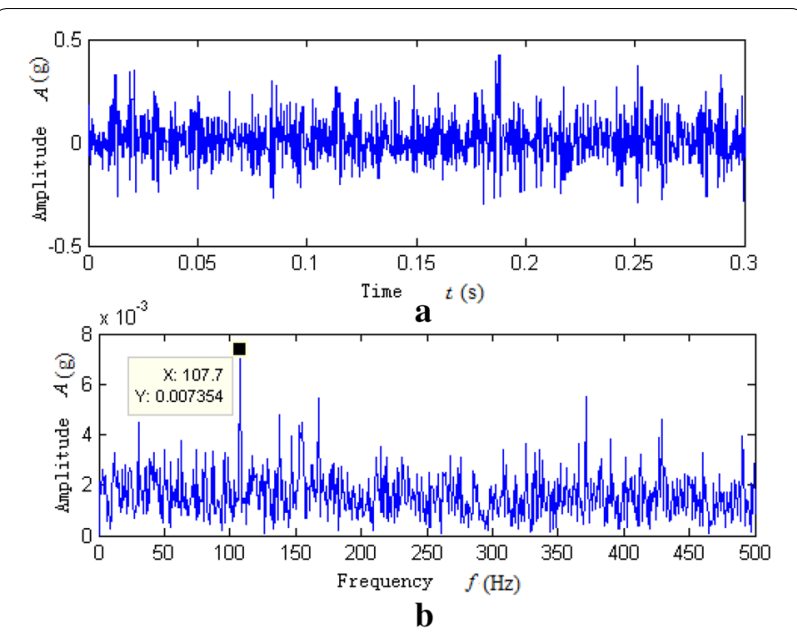

Figure 22 Filter result of 6205 with the slight defect on the outer race: (a) time waveform, (b) local spectrum

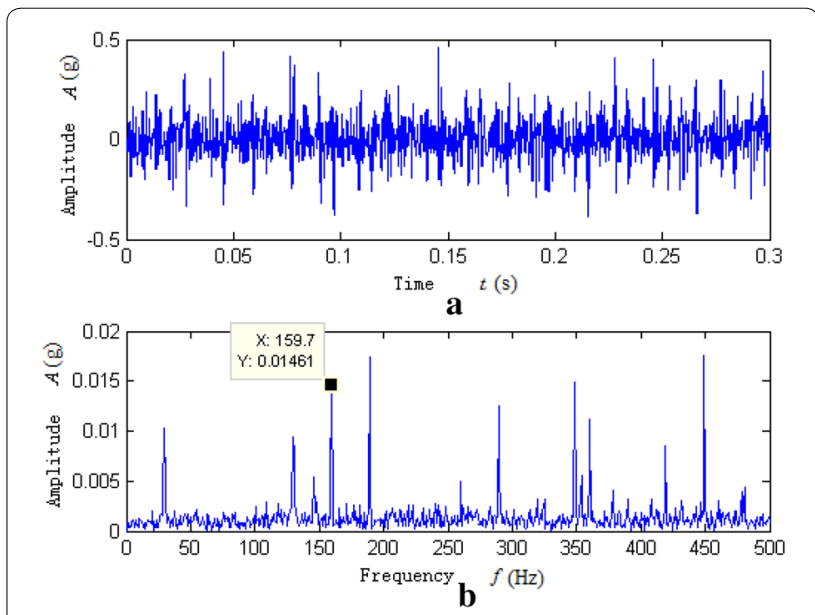

Figure 23 Filter result of 6205 with the slight defect on the inner race: (a) time waveform, (b) local spectrum

and Figure 23. From Figure 22 it is observed that the characteristic frequency (BPFO) and the rotational speed frequency are clearly visible in the spectrum. The amplitude of the BPFO is increased about five times of that of the original signal. The feature of bearing outer race fault is enhanced. Figure 23, the CMF processing result of the inner race fault signal, indicates that both the BPFI and its sidebands are all clearly detected. The lost side frequency BPFI-Fr is recovered. The modulation feature of the bearing inner race fault is enhanced. Combining the above simulation results it is established that the amplitude of the sideband frequency, even the resonant frequency in the spectrum can be affected (increased or decreased) by the interaction of the bearing fault signal. 
Since frequency loss can occur in high frequency band, the interaction of the bearing fault signal should also be considered in the frequency band selection based demodulation method.

\section{Conclusions}

This paper focuses on the interaction of the bearing vibration signal. It is presented that the phenomenon of frequency loss is generated by the internal vibrations rather than the external interfering. Multi-resonant response is excited when rolling elements strike a local fault. The harmonics in the Fourier transform spectrum of the collected vibration signal is the vector sum of that of each response. The interaction of the multi-resonant response leads to frequency loss and distortion in the spectrum. Frequency loss can be found in the low frequency harmonic, the sideband frequency and the resonant frequency. Theoretical and practical analysis demonstrates the existence of the frequency loss in bearing signal. Since frequency loss can occur in side band frequency and the resonant frequency, the interaction of the bearing fault signal should be considered in the frequency band selection based demodulation method. Based on morphological filter, a new method is provided to recover the lost frequencies. Compared with the traditional digital filter, the property of CMF in impulsive type signal processing is analyzed. The simulation and experiment results show that the morphological filter can effectively separate the interaction of bearing signal and enhance the bearing fault feature.

\section{Authors' Contributions}

AH was in charge of the whole trial; $\mathrm{AH}, \mathrm{LX}$ and SX wrote the manuscript; SX assisted with sampling and laboratory analyses. All authors read and approved the final manuscript.

\section{Authors' Information \\ Aijun Hu, born in 1971, is currently a professor at Department of Mechanical Engineering, North China Electric Power University, China. He received his PhD degree from North China Electric Power University, China, in 2008. His research interests are signal process, condition monitoring and fault diagnosis. \\ Ling Xiang, born in 1971, is currently a professor at Department of Mechani- cal Engineering, North China Electric Power University, China. She received her PhD degree from North China Electric Power University, China. Her research interests include, condition monitoring and fault diagnosis. \\ Sha Xu, born in 1989, is currently a master candidate at Department of Mechanical Engineering, North China Electric Power University, China. \\ Jianfeng Lin, born in 1991, he received his master degree from North China Electric Power University, China, in 2018.}

\section{Competing Interests}

The authors declare that they have no competing interests.

\section{Funding}

Supported by National Natural Science Foundation of China (Grant Nos. 51675178, 51475164).

Received: 27 May 2017 Accepted: 5 April 2019

Published online: 15 April 2019

\section{References}

[1] W R Thomas, A B Francisco, M V Flávio. Heterogeneous feature models and feature selection applied to bearing fault diagnosis. IEEE Transactions on Industrial Electronics, 2015, 62(1): 637-646.

[2] J Yu. Local and nonlocal preserving projection for bearing defect classification and performance assessment. IEEE Transactions on Industrial Electronics, 2012, 59(5): 2363-2376.

[3] L Barbini, M Eltabach, A J Hillis, et al. Amplitude-cyclic frequency decomposition of vibration signals for bearing fault diagnosis based on phase editing. Mechanical Systems and Signal Processing, 2018, 103: 76-88.

[4] D Zhao, J Li, W Cheng, et al. Compound faults detection of rolling element bearing based on the generalized demodulation algorithm under time-varying rotational speed. Journal of Sound and Vibration, 2016, 378: 109-123.

[5] I Antoniadis, G Glossiotis. Cyclostationary analysis of rolling-element bearing vibration signals. Journal of Sound and Vibration, 2001, 248(5): 829-845.

[6] J Yuan, Y Wang, Y Peng, et al. Weak fault detection and health degradation monitoring using customized standard multiwavelets. Mechanical Systems and Signal Processing, 2017, 94: 384-399.

[7] Jinglong Chen, Jun Pan, Zipeng Li, et al. Generator bearing fault diagnosis for wind turbine via empirical wavelet transform using measured vibration signals. Renewable Energy, 2016, 89: 80-92.

[8] L Wang, Z Liu, Q Miao, et al. Time-frequency analysis based on ensemble local mean decomposition and fast kurtogram for rotating machinery fault diagnosis. Mechanical Systems and Signal Processing, 2018, 103: 60-75.

[9] C Mishra, A K Samantaray, G Chakraborty. Ball bearing defect models: A study of simulated and experimental fault signatures. Journal of Sound and Vibration, 2017, 400: 86-112.

[10] K Sidra, J K Dutt, N Tandon. Extracting rolling element bearing faults from noisy vibration signal using Kalman filter. ASME J. Vibration and Acoustics, 2014, 136: 031008.

[11] R B Randall, J Antoni. Rolling element bearing diagnostics-A tutorial. Mechanical Systems and Signal Processing, 2011, 25(2): 485-520.

[12] M C Pan, W C Tsao. Using appropriate IMFs for envelope analysis in multiple fault diagnosis of ball bearings. International Journal of Mechanical Sciences, 2013, 69: 114-124.

[13] A Siddique, G S Yadava, B Singh. A review of stator fault monitoring techniques of induction motors. IEEE Trans. Energy Convers, 2005, 20(1): 106-114.

[14] Y X Wang, M Liang. An adaptive SK technique and its application for fault detection of rolling element bearings. Mechanical Systems and Signal Processing, 2011, 25(5): 1750-1764.

[15] J Zarei, M A Tajeddini, H R Karimi. Vibration analysis for bearing fault detection and classification using an intelligent filter. Mechatronic, 2014, 24(2): 151-157.

[16] N Sawalhi, R B Randall. Simulating gear and bearing interactions in the presence of faults_Part II__Simulation of the vibrations produced by extended bearing faults. Mechanical Systems and Signal Processing, 2008, 22(8): 1952-1966.

[17] Yonghao Miao, Ming Zhao, Jing Lin, et al. Application of an improved maximum correlated kurtosis deconvolution method for fault diagnosis of rolling element bearings, Mechanical Systems and Signal Processing, 2017, 92: 173-195.

[18] The infogram: Entropic evidence of the signature of repetitive transients. Mechanical Systems and Signal Processing, 2016, 74: 73-94.

[19] Zhipeng Feng, Haoqun Ma, Ming J Zuo. Spectral negentropy based sidebands and demodulation analysis for planet bearing fault diagnosis. Journal of Sound and Vibration, 2017, 410: 124-150.

[20] Bearing Data Center, Case Western Reserve Univ., Cleveland, OH. [Online]. Available: http://www.eecs.case.edu/laboratory/bearing.

[21] N Sawalhi, R B Randall. Vibration response of spalled rolling element bearings: Observations, simulations and signal processing techniques to track the spall size. Mechanical Systems and Signal Processing, 2011, 25(3): 846-870.

[22] P Maragos, RW Schafer. Morphological fi1ters -Part I: Their set theoretic analysis and relation to linear shift invariant filters. IEEE Transactions on Acoustics, Speech and Signal Processing, 1987, 35(8): 1153-1169. 
[23] P Maragos, RW Schafer. Morphological fi1ters -Part II: Their relation to median, order-statistic, and stack filters. IEEE Transactions on Acoustics, Speech and Signal Processing, 1987, 35(8): 1170-1184

[24] J Lv, J Yu. Average combination difference morphological filters for fault feature extraction of bearing. Mechanical Systems and Signal Processing, 2018, 100: 827-845.

[25] Y Li, X Liang, J Lin, et al. Train axle bearing fault detection using a feature selection scheme based multi-scale morphological filter. Mechanical Systems and Signal Processing, 2018, 101: 435-448.

[26] D Yu, M Wang, X Cheng. A method for the compound fault diagnosis of gearboxes based on morphological component analysis. Measurement, 2016, 91: 519-531.
[27] A J Hu, L Xiang. Selection principle of mathematical morphological operators in vibration signal processing. Journal of Vibration and Control, 2016, 22(14): 3157-3168.

[28] Antoni. Fast computation of the kurtogram for the detection of transient faults. Mechanical Systems and Signal Processing, 2007, 21(1): 108-124.

[29] A Garcia-Perez, R J Romero-Troncoso, E Cabal-Yepez, et al. The application of high-resolution spectral analysis for identifying multiple combined faults in induction motors. IEEE Transactions on Industrial Electronics, 2011, 58(5): 2002-2010.

\section{Submit your manuscript to a SpringerOpen ${ }^{\odot}$ journal and benefit from:}

- Convenient online submission

- Rigorous peer review

- Open access: articles freely available online

- High visibility within the field

- Retaining the copyright to your article

Submit your next manuscript at $\boldsymbol{s p r i n g e r o p e n . c o m ~}$ 